\title{
NUMERICAL ANALYSIS OF INFILTRATION IN A DRAINED BEACH
}

\author{
A. SAPONIERI \& L. DAMIANI \\ Dipartimento di Ingegneria Civile, Ambientale, del Territorio, Edile e di Chimica, Politecnico di Bari, Italy.
}

\begin{abstract}
The beach drainage system (BDS) is a 'soft' engineering solution for coastal protection and management. It artificially increases beach permeability by inducing the groundwater table lowering and, consequently, it favours the infiltration of sea water inside the beach. The new hydrodynamic condition would produce a reduction of the offshore sediment transport and the settlement of the suspended load. The present work focuses on a numerical simulation of infiltration processes in drained conditions by using the HYDRUS-2D code. The groundwater flow through a partially saturated porous media is described by using the well-known Richard's equation. Numerical results are then compared with experimental ones obtained during an intensive experimental program performed at the Grosser Wellen-Kanal in Hannover on a BDS prototype. Moreover, different beach permeabilities (ranging from silt to coarse sand) and different drain altimetric positions are numerically simulated. These analyses allow to draw some first considerations about BDS design criteria. In particular, a 'limit drain depth', strictly related to beach permeability, is defined as the maximum depth that produces the maximum water table lowering and, as a consequence, the best system efficacy in drying the porous medium. Keywords: BDS design, beach drainage system, coastal management, infiltration.
\end{abstract}

\section{INTRODUCTION}

Coastal areas constitute high-energy zones characterized by an active and complex dynamics, in a delicate equilibrium, susceptible to a considerable instability due to both natural evolution trends and man-induced processes. The causes of such instability mainly refer to the decreasing of natural sand supply.

Coastal erosion is a process characterized by a complex historical and geographical background that makes difficult to solve the land-use problems by the traditional coastal protection works. There is a delicate balance between the requirement of primary protection against erosion on one hand, and the protection of dynamic coastal landscape on the other hand. Historically, protective measures have been reactive in nature and concentrated on preventing sand loss, resulting in a serious impact on both hydrodynamics and morphodynamics and, hence, on the unaltered coastal ecosystems close to the protected area. Especially in the presence of hard defence structures, the protection of one area is generally at the expanse of increased erosion downward from the protected area, with a consequent geographic shift of the erosion [1]. Soft solutions, as the artificial beach nourishment, have a less impact on shore dynamics. Such a solution is environmentally acceptable but needs to be carefully designed. Usually, nourished beach required periodic sand recharges that allow to maintain the equilibrium of the new beach profile.

The beach drainage system (BDS) can be numbered among soft engineering systems for coastal protection. In general, the system artificially increases the sand permeability, producing a groundwater table lowering and an increase in the thickness of the unsaturated zone inside the beach. It aims to interact with the swash hydrodynamics by favouring the deposition of sediments transported by waves during the uprush phase and contrasting their offshore movement during the backwash. Both field and laboratory experiences on the system response show that the effective beach stabilization is still not well defined. Performed studies point out that the system is not able to totally restore an eroded sandy profile, demonstrating slight effects in reducing beach erosion. A sand volume accretion 
can be observed close to the zone where the drain is placed. For this reason, the BDS can be considered as an auxiliary system for the management of the soft solutions, as the artificial nourishment. In addition to all traditional coastal protection systems, the BDS has to be supported by a continuous beach monitoring and management. To date, different studies are conducted aimed to extend the life of the artificial nourishment, resulting in a reduction of periodic filling and, hence, of the management costs. Recently, the so-called 'protected' nourishment is substituting the traditional 'free' nourishment by the adoption of retaining groynes or breakwaters, or, as proposed above, by using a BDS.

In literature, several works on BDS field installations around the world are available [2-5], showing different systems' responses in reducing offshore sand transport. The analysis of field installations remarks the lack of a standard methodology for the drainage system design. The lack of data, due to a discontinuous long-term field monitoring and to the irregular system working, (generally related to administrative problems and permanent damages) and the few performed laboratory experiments cause several uncertainties about working mechanisms, and the involved hydrodynamic and morphodynamic processes. In these conditions, the study of drainage effects results to be very difficult.

As a matter of fact, the system efficacy in reducing beach erosion was discovered 'by chance' in Denmark, without previous laboratory tests or theoretical studies. In order to define the system applicability limits and its main design parameters (i.e. number of pipes, pipe diameter, excavation depth, altimetric and planimetric position), both monitored BDS field installation and laboratory experiments under known boundary and initial conditions are needed.

As previously specified, the BDS efficacy is strictly related to the interaction between the external wave motion and the groundwater inside the beach. Indeed, in such coastal engineering problems, one of the main pursued aims is to correctly reproduce and predict the water table variations inside the beach as a function of the wave run-up/down. The study of filtration processes inside a partially saturated porous medium represents the first step in order to develop such a kind of models. To date, the BDS design is only based on the acquired experiences on field installations and empirical formula. For this reason, laboratory experiments have to be combined with numerical modelling in order to extrapolate information from a limited number of field and laboratory experiments to different drain configurations, wave climate and beach characteristics.

After a brief discussion on the physical principles, which drive the BDS working as a coastal defence measure, the present work deals with a 2D numerical analysis of the BDS effects on the groundwater behaviour inside a beach, by using the HYDRUS-2D code that numerically solves the Richard's equations in a partially saturated porous medium. Laboratory static conditions are numerically simulated in order to obtain more information about system design criteria. Measured and numerical data are compared and then the influence of the main drainage design parameters (i.e. drain diameter, position, sand permeability, etc.) on water table lowering drainage efficiency is discussed. Experimental data used in the present work were obtained during an intensive program of laboratory tests carried out on a full-scale BDS model at the Grosser Wellen-Kanal of Hannover University (Germany) in 2009.

\section{THE BEACH DRAINAGE SYSTEM}

\subsection{The physical process}

In general, the BDS produces an increase of the beach permeability due to water table artificial lowering, with a consequent increase in the thickness of the unsaturated zone inside the beach. 
One of the most important causes affecting the cross shore sediment transport is the water infiltration inside the beach through the shore face, mainly related to the hydrodynamic conditions during uprush and backwash [6,7], and sand characteristics [8] (i.e. permeability, saturation degree, etc.). In particular, Turner and Masselink [9] found that the in/ex filtration processes in the swash zone can increase the sediment transport rate by up to $40 \%$ of the peak transport rate during the uprush and decrease it by $10 \%$ during backwash.

Both field measurements and numerical modelling demonstrate that the near shore groundwater dynamics is principally driven by tides, waves and their interactions with the beach morphology [10].

Grant [11,12] first suggested a link between beach groundwater behaviour and swash zone sediment transport, strictly related to the relative elevation of the beach groundwater table with respect to the external mean sea level.

When the beach is interested by long-period waves, the relative low water table (Fig. 1a) enhances the swash infiltration and, as a consequence, the settlement of the suspended sediments. The run-up flow can infiltrate inside the porous medium, causing an increase in the effective stresses and, thus, of the sediment stability. On the contrary, when the beach is interested by winter storms (characterized by higher energy and shorter period waves), the high water table (Fig. 1b) promotes the ex-filtration process. The backwash flux prevails on the uprush one because the water cannot infiltrate through the sand and flushes down due to the high saturation degree of the beach. The net result of the run-up/down processes is an offshore sediment transport causing, consequently, the shore erosion. If the sand is partially saturated, the beach behaviour can be compared with the summer coastal dynamics with a visible advance of the shoreline. Conversely, if the saturation degree of the beach is close to the unit, the beach assumes a typical winter behaviour with sediments eroded from the shore zone and moved offshore.

The relevance of these processes for beach profile development was effectively demonstrated by John and Duncan [13], discovering cut-and-fill cycles on tidal beaches. As early as the 1970s [14], laboratory studies showed that it is possible to enhance the sediment stability across the swash zone by artificially lowering the groundwater level. Kawata and Tsuchiya [15] tested in laboratory the efficiency of a sub-sand system as a system of beach erosion control, under controlled conditions. According to obtained results, the authors showed the applicability of the drainage system in order to prevent or mitigate beach erosion under any wave conditions. Under swell waves, the sub-sand filter allowed to accelerate the sediment accretion in the foreshore zone through the development of a berm with a consequent shoreline advance. When wave conditions changed to stormy waves the decreasing of sediment loss from the foreshore was observed. Oh and Dean [16] performed laboratory studies at the Coastal and Oceanographical Engineering Laboratory in order to investigate the effects of groundwater table elevations on the beach profile changes over the swash zone. They

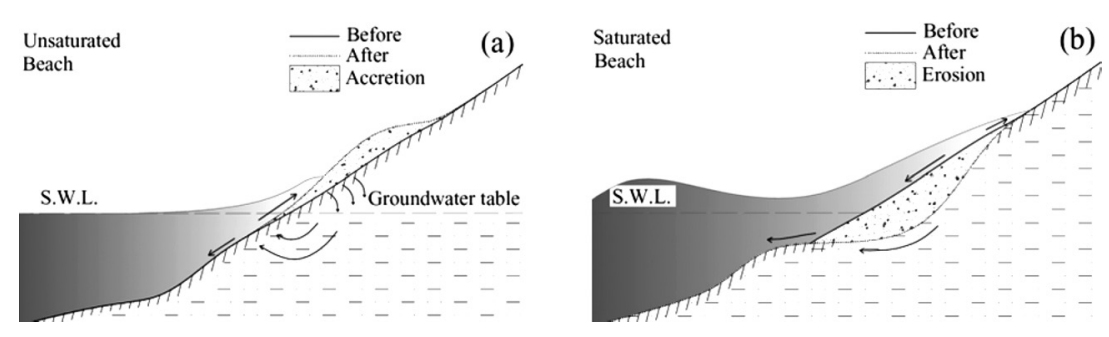

Figure 1: Unsaturated (a) and saturated (b) beach behaviour. 
investigated the changes of an initially linear profile subject to regular wave at three different water table levels, strengthening the influence of beach water tables on profile dynamics.

Masselink et al. [17] performed large-scale laboratory tests (Delta flume, Netherlands) in order to investigate the effects of the beach groundwater table on gravel beach morphology. Different ranges of wave and water-level conditions were tested. Experiments demonstrated that the onshore netsediment transport was most pronounced with a steady sea level and long-period waves, while the swash zone erosion was observed with elevating the groundwater table.

\subsection{The BDS}

The BDS is constituted by one or more drains placed under the beach face running parallel to the shoreline. Drains consist of polyvinyl chloride (PVC) pipes with holes uniformly distributed on their contour in order to drain the water. Drains are connected through blind pipes with a collection point from where the water is pumped out and then transported back again to the sea or to another destination.

As already discussed in Section 1, several BDS field applications are available around the world. Even if field monitoring is very useful to assess the effectiveness of the system [18], laboratory tests can be used in order to clarify the system response under different wave conditions. For this purpose, 1:1 scaled experiments were undertaken at the Grosser Wellen-Kanal (GWK) of Hannover University (Germany) in 2009/2010. The projects involved a series of experiments aimed to test the system response under different wave conditions and with different system configurations.

In the following, the GWK large-scale model is briefly described. Obtained results on water table lowering and drained flows are presented in order to validate the application of the numerical simulations performed by using the HYDRUS-2D code.

\section{THE PHYSICAL MODEL}

The laboratory investigation on a full-scale physical model of the BDS was performed at the Coastal Research Centre (FZK) of Technical University of Brounischweig in Hannover (Germany). Figure 2 shows the beach profile realized at the laboratory.

Beach profile consisted of almost uniform quartzitic sand, with an average diameter $\left(\mathrm{d}_{50}\right)$ equal to $0.33 \mathrm{~mm}$. Laboratory sand measurements performed at the Politecnicodi Bari (Italy [19]) show that the sand had also a small percentage of fine fractions due to water derivation from a river near the laboratory, although it was filtered before entering into the channel. To analyse the filtration processes inside the beach, the average fall velocity and the coefficient of permeability were measured and founded to be equal to 4.8 and $3.2 \times 10^{3} \mathrm{~cm} / \mathrm{s}$.

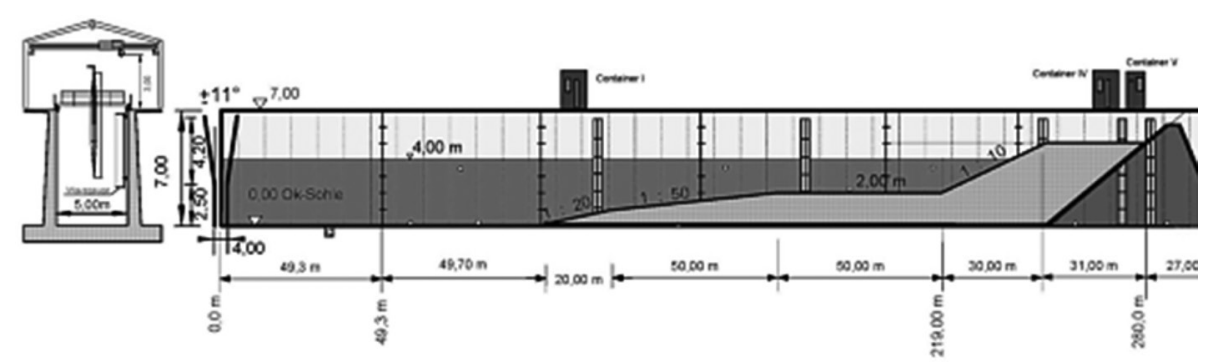

Figure 2: Sketch of GWK wave flume with sandy beach profile. 
a)
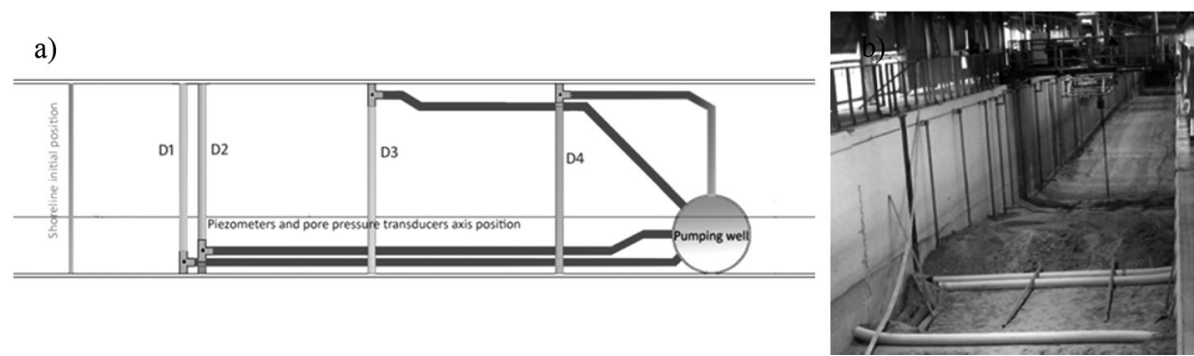

Figure 3: (a) Plain view of the drainage system and (b) installation of the drains.

Table 1: Drains position.

\begin{tabular}{lccc}
\hline & $x$ & $Y$ & $\begin{array}{c}\text { Distance from the } \\
\text { initial shoreline } \\
\text { Drain }\end{array}$ \\
\hline D1 & 242.10 & 3.46 & 2.60 \\
D2 & 242.40 & 3.47 & 2.90 \\
D3 & 247.00 & 3.45 & 7.50 \\
D4 & 252.00 & 3.45 & 12.50 \\
\hline
\end{tabular}

The adopted drainage system (Fig. 3a and b) was constituted by four corrugated PVC pipes installed inside the beach, below the static water level (SWL) (at $3.6 \mathrm{~m}$ from the flume bottom), in correspondence to the swash zone and transversally to the incident wave motion at different distances from the shoreline (Table 1).

Variable spatial drain positions were considered in order to analyse the optimal distance from the shoreline in terms of morphodynamic and hydrodynamic efficiency. As it can be observed, the distance between the first two drains is smaller with respect to the other ones. They were placed side by side in the same hole inside the sand. Such a configuration gave the possibility to simulate a drain with a double diameter (by opening the two drains at the same time) in order to study the influence of drain dimensions on BDS efficiency in draining water flows. Each drain had a diameter equal to $0.20 \mathrm{~m}$ and it was 5-m long, occupying the entire beach width. Moreover, drains were covered by a double layer of geotextile in order to avoid the blockage due to the sand during water drainage with a probably out of action of the system. Drained water was then transported to a pumping well through PVC pipes and finally discharged into the flume by means of an external iron pipe. The end of each pipe, inside the pumping well, was provided with a gate valve to allow the drains to be switched on/off.

Two sets of tests were performed. The first one (15 static tests) with two different undisturbed sea water levels equal to 4.00 and $4.20 \mathrm{~m}$, respectively. The higher water level enables to simulate the wave-induced set-up due to the sea-tide. During the second set of tests (64 dynamic tests), drainage system was tested under different wave energy conditions. Three kinds of irregular wave attacks characterized by a JONSWAP spectrum were performed in order to reproduce high, medium and low wave energy conditions. The duration of tests was set to achieve a quasi-stationary condition.

During the tests, both morphodynamic and hydrodynamic behaviours of drained beach were analysed by using the data sampled by a large number of instruments. Surface elevation, pressure, velocity, drainage flow and beach profile were measured in order to delve into the nearshore 
processes. A detailed description of the performed experiments, measurements method and results can be found in previous works [20-24].

In the present work, only water table oscillations and drained flows are reported. A set of 12 piezometers and 10 pore pressure transducers were installed along the beach profile in order to measure the water table fluctuations during the tests. Each pressure cell was placed at about the same depth of drains and at about $2 \mathrm{~m}$ from the left wall of the flume with respect to the wave generator (Fig. 3a). Other four pore pressure transducers were located inside the terminal part of each drain. These transducers were used in order to study the hydraulic regime of the drainage system, by measuring the hydraulic head inside the pipe.

The drainage discharges were evaluated using both a flowmeter and a pore pressure transducer. The flowmeter was located on the external pipe used for drained water discharge. The pore pressure transducer was placed in the pumping well at $0.08 \mathrm{~m}$ from the bottom.

\section{THE NUMERICAL MODEL}

\subsection{Literature overview}

The study of infiltration processes inside a partially saturated porous medium represents the first step in order to develop such models to be used in Beach Dewatering System design.

In literature, several models have been proposed in order to predict the wave-induced run-up and to study the water table variations inside a beach. Unfortunately, these models need complex adjustments if the analysed beach is equipped with a drainage system. Few models have been developed in order to simulate hydrodynamic and morphodynamic processes in the presence of a drain. Moreover, the research is also limited by few available data and laboratory experiments, which would allow to validate proposed mathematical models. Packwood [25] improved a model that analyses the influence of sand porosity on uprush flow due to a single tide wave on a gently sloped beach initially unsaturated. The model focuses on the fact that the sediment transport on beach face is strictly related to the infiltration processes in the swash zone. Sand permeability constitutes one of the main characteristics influencing the infiltrated flow rate. Indeed, experimental results show that a fine sandy beach could be compared to an impermeable one, with respect to the infiltration process. If beach is composed of medium coarse sand, the backwash flux is highly reduced because a greater flux can infiltrate inside beach during run-up phase. The filtration process is described by using Dicker's equation under the assumption of hydrostatic pressure distribution along the submerged beach and filtration prevailing in vertical direction. The author describes the uprush on beach face by using the shallow water equations. Results show that for a medium-fine sand, the effects of the porosity on the uprush flux resulted to be less relevant than on the backwash phase. Moreover, later laboratory experiments [26] demonstrated that the maximum run-up height on beach is not influenced by infiltration flow rate, while the backwash is highly reduced when the sand has a high permeability. Kobayashi and Wurjanto [27] proposed a numerical model able to simulate the uprush flow on rough beaches and the infiltration process under a perpendicular waves train. An energy equation is added to both mass and continuity equations in order to evaluate the dissipated energy rate due to the breaking. Nielsen [28] focused his studies on the water table variations inside the beach. He found that the water table position with reference to the mean water level of the sea influences many aspects of engineering projects, i.e. the structures stability or the use of agricultural soils because of the salt water intrusion. The aim of the study was the evaluation of the water table variation as a function of tidal sea fluctuations. Li et al. [29,30] defined a two-dimensional numerical model aimed to simulate the behaviour of a coastal aquifer under the influence of a drainage system. 
The involved processes are described by using Darcy's law, continuity and Laplace's equations by imposing the appropriate boundary conditions. Results obtained by numerical simulations showed a good agreement compared with field data. Successively, Vesterby also focused his studies on the numerical simulation of a BDS [31] in order to simulate hydrodynamic processes inside the beach and to evaluate the water table variations. The numerical model is improved by using the hydrological model MIKE SHE for the filtration process and the model MIKE11 for the hydrodynamic of the external wave motion in the swash zone. Li et al. [32] derived the numerical model called Beachwin. It represents an evolution of the previous work performed by the authors [29,30]. The model allows to simulate separately the involved phenomena: the wave propagation on the beach, the filtration processes in the unsaturated zone, the sediment transport in the shore-zone and the shoreline evolution. The model solves the Laplace equations for a saturated porous medium. Recently, Ioannidis and Karambas [33] have developed another conceptual model for simulating the variation of the saturation line inside the beach with the presence of a drainage system. The author describes the external wave motion by using the Boussinesq equations referring to van Gent work mentioned above. The long wave equations are used to describe the porous flow model. Model has been validated with field data and results are quite satisfactory.

In the present work, groundwater flow has been simulated by using Richard's equations, numerically solved by the HYDRUS-2D code and described in the following sections.

\subsection{The mathematical model}

The HYDRUS-2D software package simulates two-dimensional water flow, heat movement and transport of solutes involved in sequential first-order decay reactions in unsaturated, partially saturated or fully saturated porous media. The code can handle flow domains delineated by irregular boundaries and the flow region may be composed of nonuniform soils having an arbitrary degree of local anisotropy. Saturated-unsaturated water flow and convection-dispersion type equations for heat and solute transport are described by using Richard's equation. The code numerically solves the Richard's equation using Galerkin-type linear finite element schemes, including a Marquardt-Levenberg parameter optimization algorithm for inverse estimation of soil hydraulic and solute transport and reaction parameters from measured transient or steady-state flow.

Considering two-dimensional isothermal Darcian water flow in a partially saturated rigid porous medium and assuming that the air phase can be neglected in the liquid flow process, the governing equation is given by the following modified form of Richard's equation (eqn 1):

$$
\frac{\partial \vartheta}{\partial t}=\frac{\partial}{\partial x_{i}}\left[K\left(K_{i j}^{A} \frac{\partial h}{\partial x_{j}}+K_{i 2}^{A}\right)\right]-S
$$

where $\vartheta$ is the volumetric water content $\left(\mathrm{L}^{3} \mathrm{~L}^{3}\right), t$ is the time $(\mathrm{T}), h$ is the pressure head $(\mathrm{L}), K_{i j}{ }^{A}$ are components of a dimensionless anisotropy tensor $\boldsymbol{K}^{A}$ in which the diagonal of entries is equal TO one and the off-diagonal entries are zero for an isotropic medium, $x_{i}\left(i=1,2\right.$; with $x_{2}=z$ being the vertical coordinate positive upwards) are the spatial coordinates (L), $S$ is a sink term $\left(\mathrm{T}^{1}\right)$, which represents the volume of water removed per unit time from a unit volume of soil due to plant water uptake [34,35] and $K$ is the unsaturated hydraulic conductivity function $\left[\mathrm{L} \mathrm{T}^{1}\right]$ given by eqn (2):

$$
K(h, x, z)=K_{s}(x, z) K_{r}(h, x, z)
$$

where $K_{s}$ and $K_{r}$ are the saturated and the relative hydraulic conductivity, respectively. 
In the present work, the beach sand has been assumed isotropic, which means that $K_{i j}$ survives only for situation when $i=j$ and zero otherwise. Further, the sink term $S$ has been considered equal to zero. As a consequence, eqn (1) can be written as (eqn 3):

$$
\frac{\partial \vartheta}{\partial t}=\frac{\partial}{\partial x_{i}}\left[K\left(K_{i i}^{A} \frac{\partial h}{\partial x_{i}}+K_{i 2}^{A}\right)\right]
$$

The unsaturated soil hydraulic properties $\vartheta(h)$ and $K(h)$ in eqn $(1)$ are in general highly nonlinear functions of the pressure head. The expressions adopted in the present work are those of van Genuchten [36] (eqns 4 and 5):

$$
\begin{gathered}
\vartheta(h)=\left\{\begin{array}{lr}
\vartheta_{r}+\frac{\vartheta_{s}-\vartheta_{r}}{\left[1+|a h|^{n}\right]^{m}} & \text { if } h<0 \\
\vartheta_{s} & \text { if } h \geq 0
\end{array}\right\} \\
K(\vartheta)=\left\{\begin{array}{ll}
K_{s} S_{e}^{l}\left[1-\left(1-S_{e}^{1 / m}\right)^{m}\right]^{2} & \text { if } h<0 \\
K_{s} & \text { if } h \geq 0
\end{array}\right\}
\end{gathered}
$$

where $S_{e}$ is the effective water content defined as (eqn (6))

$$
S_{e}=\frac{\vartheta-\vartheta_{r}}{\vartheta_{s}-\vartheta_{r}}
$$

where ${ }_{r}$ and ${ }_{s}$ denote the residual and the saturated water contents, respectively, and , $n, m(=11 / n)$ and $l$ are empirical parameters.

Equations (4) and (5) contain six unknown independent parameters:, , $n, l$ and $K_{s}$, and $K_{s}$ have a clear physical meaning, whereas, $n$ and $l$ are empirical parameters determining the shape of the retention and hydraulic conductivity functions [36]. In particular, the pore connectivity parameter $l$ has been estimated to be about 0.5 as an average for many soils [37].

The solution of eqn (1) requires the knowledge of the initial distribution of the pressure head within the flow domain (eqn 7):

$$
h(\mathbf{x}, t)=h_{0}(\mathbf{x}) \text { for } t=0
$$

where $h_{0}$ is a prescribed function of $\mathbf{x}$.

The HYDRUS-2D code implements three different types of boundary conditions to describe system-independent interactions along the boundaries of the flow region. Therefore, eqn (1) can be numerically solved by specifying pressure head (Dirichlet type, eqn 8), flux (Neumann type, eqn 9) and gradient boundary conditions (eqn 10) given by the following functions, respectively:

$$
\begin{gathered}
h(\mathbf{x}, t)=\psi(x, t) \quad \text { for }(\mathbf{x}) \in \Gamma_{\mathrm{D}} \\
-\left[K\left(K_{i j}^{A} \frac{\partial h}{\partial x_{j}}+K_{i 2}^{A}\right)\right] n_{i}=\sigma_{1}(\mathbf{x}, \mathrm{t}) \quad \text { for }(\mathbf{x}) \in \Gamma_{\mathrm{D}}
\end{gathered}
$$




$$
\left(K_{i j}^{A} \frac{\partial h}{\partial x_{j}}+K_{i 2}^{A}\right) n_{i}=\sigma_{2}(\mathbf{x}, \mathrm{t}) \quad \text { for }(\mathbf{x}) \in \Gamma_{\mathrm{D}}
$$

where, and indicate Dirichlet, Neumann and gradient-type boundary segments, respectively, and (L), [(LT $)^{1}$ and (nondimensional) are the prescribed functions as a function of $\boldsymbol{x}$ and $t$ and $n_{i}$ are the components of the outward unit vector normal to boundary or .

In addition, there is the possibility of imposing other three different types of system-dependent boundary conditions. These three types of dependent boundary conditions cannot be defined a priori. One of these involves soil-air interfaces that are exposed to atmospheric conditions. The potential fluid flux across these interfaces is controlled exclusively by external condition. In the absence of surface ponding, the numerical solution of eqn (1) is obtained by satisfying the following two conditions (eqns 11 and 12) [38]:

$$
\begin{gathered}
\left|\left(K\left(K_{i j}^{A} \frac{\partial h}{\partial x_{j}}+K_{i 2}^{A}\right)\right) n_{i}\right| \leq E \\
h_{A}<h_{s}
\end{gathered}
$$

where $E$ is the maximum potential rate of infiltration or evaporation under the analysed atmospheric conditions, $h$ is the pressure head at the soil surface, $h_{A}$ and $h_{S}$ are minimum and maximum pressure heads allowed under the prevailing soil conditions, respectively. In general, $h_{A}$ is determined from the equilibrium conditions between soil water and atmospheric water vapour [39], whereas $h_{S}$ is set equal to zero. The second type of system-dependent boundary condition is a seepage face through which water leaves the saturated part of the flow domain. The pressure head along a seepage face is automatically set uniformly equal to zero. Additionally, the code assumes that water leaving the saturated zone across a seepage face is immediately removed by overland flow or some other removal process. The third class of system-dependent boundary conditions concerns the drains. The code assumes that as long as a drain is located in the saturated zone, the pressure head along the drain is equal to zero. The drain acts as a pressure head sink.

The solution of the governing flow (eqn 1) is obtained by using the Galerkin finite element method with linear basis function. This method has been covered in detail by many authors, i.e. Neuman [40] or Pinder [41]. In the following, only the most pertinent steps in the solution process are given. The method is composed by the following steps:

- space discretization into a network of triangular elements;

- time discretization domain into a sequence of finite time intervals;

- numerical iterative process that continues until a satisfactory degree of convergences is obtained.

\subsection{The numerical simulation}

The present work deals with the HYDRUS-2D simulation of the water table lowering in a beach equipped with a drainage system. In this first step of numerical analysis, only static conditions have been considered in order to validate the model by using the experimental data collected during GWK experiments and to obtain some general information about BDS design criteria.

The geometry reproduces the physical model adopted for GWK tests. The finite-element mesh is constructed by dividing the flow region into triangular elements. The drains are modelled as circular 


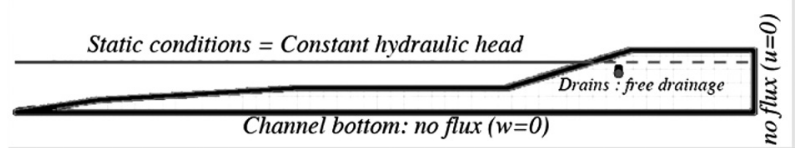

Figure 4: Mesh domain and boundary conditions used in numerical drainage simulations.

holes. The mesh is almost uniform except in the zone close to the drain where a local refinement has been used in order to obtain more accurate results, for a total number of 11,370 nodes and 21,192 triangles.

The beach is assumed homogeneous and isotropic. Running the model requires the hydraulic parameters $\vartheta_{s}, \vartheta_{r}, K_{s}, n, \alpha$ and $l$, as well as the initial water content distribution. For sand characteristics, the code allows to use some standard parameters that depend on the soil type (grain size, soil classification, etc.). The hydraulic conductivity $K_{s}$ has been set equal to that one measured in the laboratory. The initial water content distribution has not been defined a priori. In order to recreate the real laboratory initial conditions, the channel filling-up has been simulated by imposing a constant hydraulic head (equal to 4.00 and $4.20 \mathrm{~m}$, respectively) on the bottom of the beach, causing the gradual beach saturation from the bottom to the top, like as the channel filling-up reproduced during laboratory tests. The output of the hydraulic head distribution in the beach has been then used as the initial condition for the drainage processes' simulations.

Figure 4 schematically reports the adopted beach geometry, the mesh domain and the relative boundary conditions imposed for simulating the infiltration processes artificially induced by the drain working. The bottom of the beach (corresponding to the bottom of the laboratory channel, about $7 \mathrm{~m}$ under the drain) and the vertical beach contour (on the right side in Fig. 4, about $20 \mathrm{~m}$ far from the drain) have been considered no flux boundaries. This assumption derives from considering that in correspondence to the above distances from the working drain, the influence of drainage can be neglected. The drains are considered free drainage surfaces, because during experiments a free surface flows within the drains was observed. Under such an assumption, the code assumes that as long as a drain is located in the saturated zone, the pressure head along the drain is equal to zero. On the left side in Fig. 4, static conditions (with a SWL equal to $4.00 \mathrm{~m}$ for test $\mathrm{S} 1$ and $4.20 \mathrm{~m}$ for test S2) have been simulated by imposing a constant hydraulic head during the drainage processes along the submerged beach profile.

The time of each simulation has been set at $60^{\prime}$ (as for laboratory runs) and the time discretization $\Delta t$ has be set equal to 0.001. A smaller time interval $\Delta t$ would cause the bad convergence of the numerical solution.

\section{RESULTS}

Figures 5 and 6 show two examples of both experimental and analytical spatial variation of water table lowering artificially induced by the activation of the drain D2 (Fig. 5) and D3 (Fig. 6) in constant hydraulic head conditions at the end of simulation $(t=1 \mathrm{~h})$. The simulated sea water level is equal to $4.00 \mathrm{~m}$ (test S1). Moreover, the beach profile measured in laboratory is reported. Grey line represents the spatial variation of water table numerically modelled, while black circles represent the local piezometric head measured in laboratory by piezometers.

The comparison between laboratory and numerical results shows that the numerical model simulates quite well the depression cone due to the activation of drain [42]. In fact, in all tested drain 


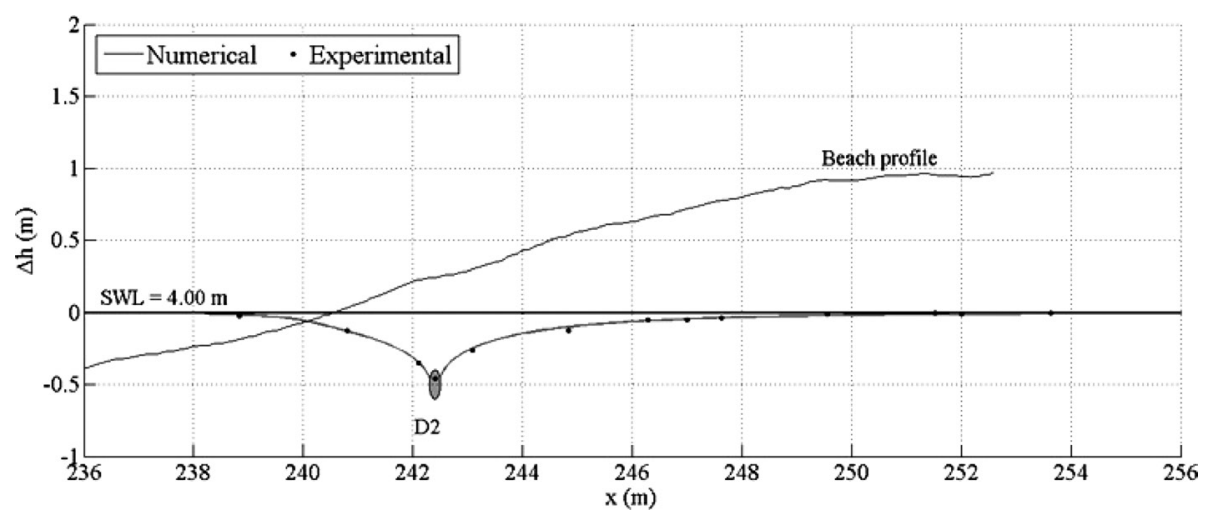

Figure 5: Comparison between numerical (-) and experimental (o) spatial variation of water table lowering (Test S1 - drain D2).

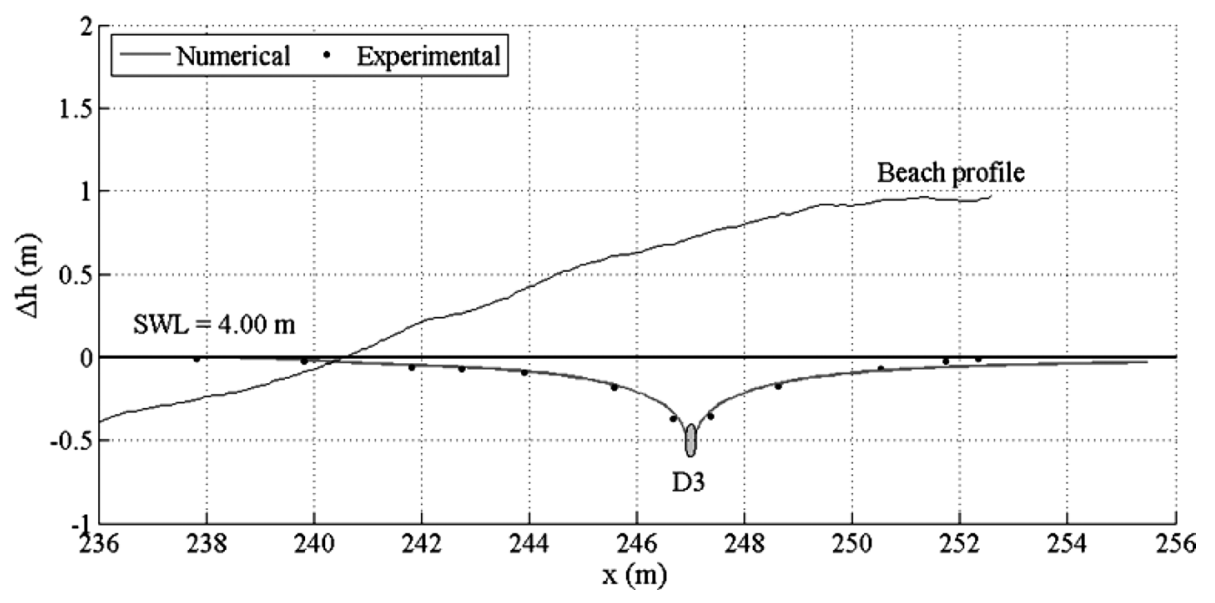

Figure 6: Comparison between numerical (-) and experimental (o) spatial variation of water table lowering (Test S1 - drain D3).

configurations, the saturation line tends to the initial undisturbed groundwater level in the inner part of the beach and to the SWL seawards and its maximum occurs in correspondence of the working drain.

The good agreement between numerical and experimental results can also be observed for tests S2, with a SWL equal to $4.20 \mathrm{~m}$. Figure 7 reports an example of both numerical and experimental spatial variation of water table lowering artificially induced by the activation of the drain D4.

The most remarkable result obtained by simulating the drainage process is the groundwater behaviour in correspondence of drain, which has not yet been deeply analysed. Figure $8 \mathrm{a}$ and $\mathrm{b}$ reports the numerical maximum water table lowering $(\Delta h)$ and the pressure head measured inside drains $\left(p_{D}\right)$ during laboratory tests for tests $\mathrm{S} 1$ and $\mathrm{S} 2$, respectively. The analysis of data shows that when the water level is equal to $4.00 \mathrm{~m}$ (Fig. 8a), a free surface flow occurs within the drain pipes and the saturation lines reach the drain contours. When the water level is higher (Fig. 8b), both groundwater and pipes behaviours are not well defined. When D4 is active, the maximum water table 


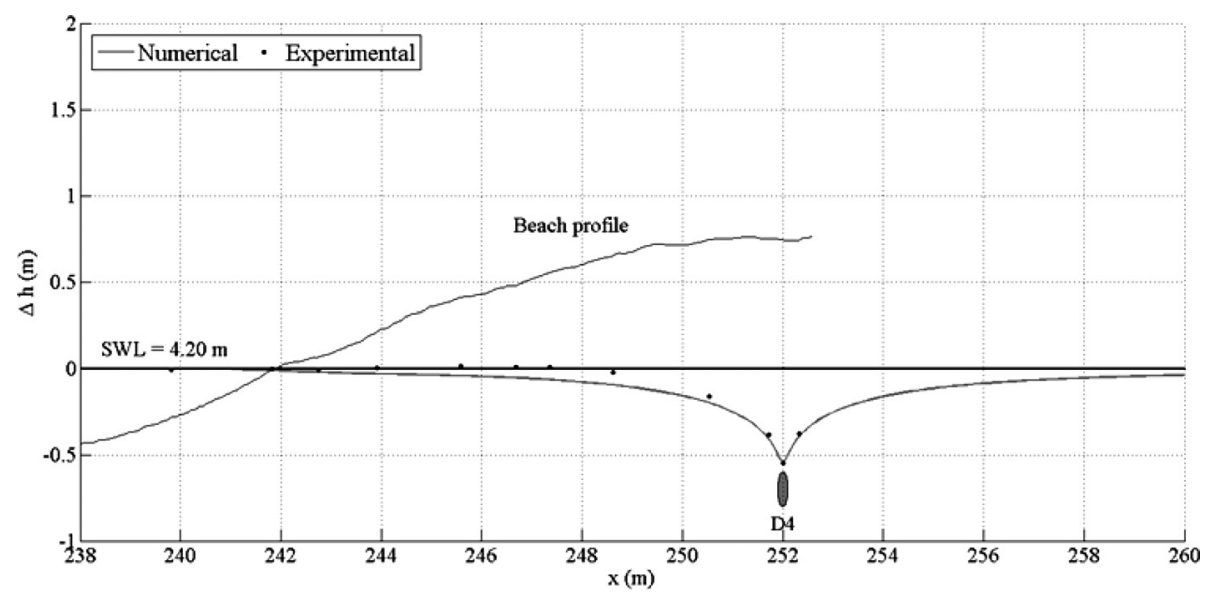

Figure 7: Comparison of numerical and experimental spatial variation of water table lowering (Test S2 - drain D4).

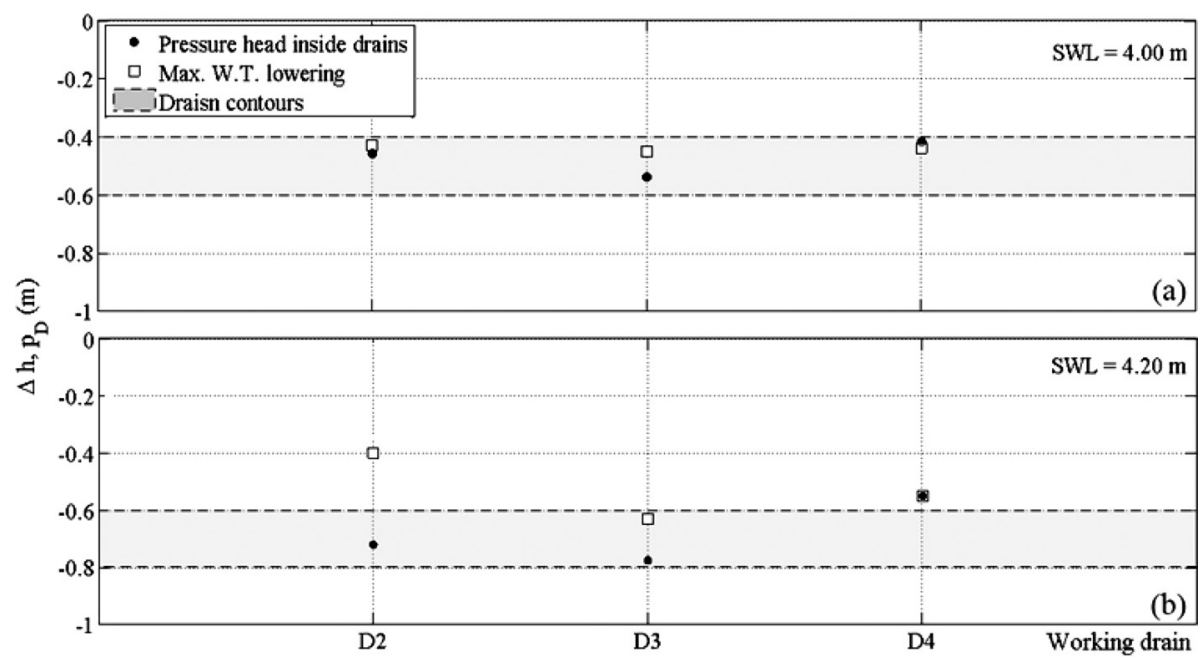

Figure 8: Maximum water table lowering $(\Delta h)$ and pressure head inside drains $\left(p_{D}\right)$ for tests $\mathrm{S} 1$ (a) and S2 (b) with single drains D2, D3 and D4.

lowering corresponds to the hydraulic head measured by the pore pressure transducer placed inside the drain, confirming that D4 works in pressure. On the contrary, a free surface flow is measured by both the pore pressure transducers placed inside the drains D2 and D3. In such a configuration, the water table behaviour is different and not well defined. In fact, the activation of D2 causes a water table lowering that reaches its maximum outside the drain, which does not correspond to the hydraulic head measured inside. When D3 is open the water table joins the pipe, showing a local piezometric head higher than that measured by the transducer inside the drain. In these two last configurations, results seem to show that two different hydraulic behaviours occur in the porous medium and within the pipe. The lack of a pressure transducer during laboratory tests placed over 


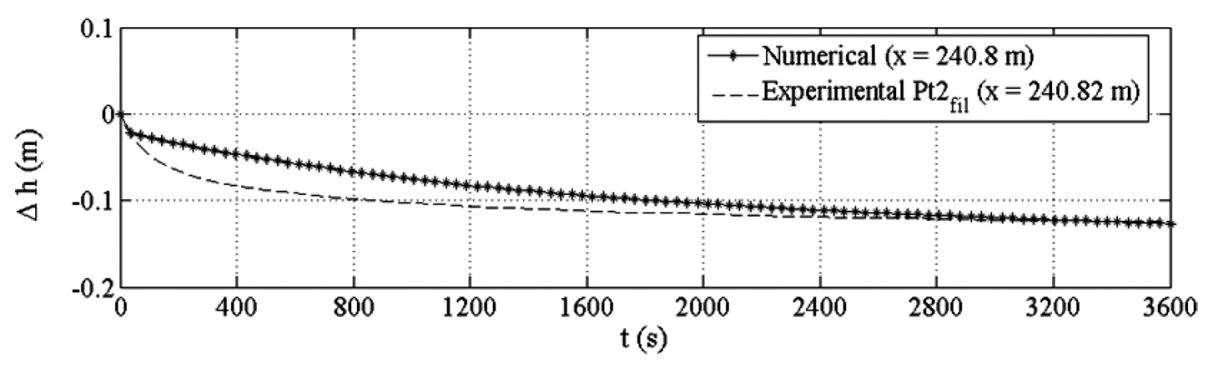

Figure 9: Measured (-) and calculated (+) time variation of water table lowering (Test S1 - drain D2).

the drain at the same section of that one placed inside does not allow to understand the real drain flow regime.

Some differences between numerical and experimental results can be found in the analysis of the time variation of water table lowering. Figure 9 reports an example of both calculated (stars) and measured (dotted line) time variation of the local piezometric head for the test S1 with the drain D2 open. The experimental data are those collected by the pore pressure transducer placed near the drain D2, at $240.8 \mathrm{~m}$ from the wave generator and at $0.5 \mathrm{~m}$ under the SWL. To compare the data, the numerical results are referred to the mesh node at the same piezometer plano-altimetric position.

Figure 9 points out that when the process reaches a quasi-steady condition, the two models show almost the same water table lowering velocity, assuming at the end the same value of hydraulic head. At the beginning of the lowering process, the transient numerically simulated by the code is more gradual than in laboratory experiments. In fact, in all tested configurations, about the $70 \div 80 \%$ of the total hydraulic head lowering was observed in the first 8-10 min reaching than the quasi-steady state. Differently, in the numerical model the transient lasts for about $30 \mathrm{~min}$. In the present work, the analysis regards the study of the water table position at the end of tests, so such differences do not influence the results that are shown in the following. For this reason, the very good agreement shown by the numerical results in simulating the drained water table lowering gives the possibility to improve other numerical simulations on BDS effects in static conditions with different system configurations and sand characteristics. In particular, different sand permeabilities and drain depths with respect to the SWL have been simulated.

The planimetric position of the drain with respect to the shoreline has been set constant for all performed simulations. Experiments show that considering both hydrodynamic and morphodynamic effects induced by the activation of the drainage system, the best efficacy can be observed when the planimetric drain position is close to the run-up limit, which during laboratory tests corresponds to the position of drain D3.

Figure 10 reports the numerical spatial variation of water table induced by the drain D3 placed at about $7.50 \mathrm{~m}$ from the shoreline, at $0.5 \mathrm{~m}$ under the SWL equal to $4.00 \mathrm{~m}$, in a beach characterized by different values of permeability (from $10^{8}$ to $10^{3} \mathrm{~m} / \mathrm{s}$ ), corresponding to typical beach grain sizes (from silt to coarse sand). As it was expected, an increase in beach permeability causes an increase in the unsaturated area within the porous medium. The saturation line referring to the maximum value of permeability (the lowest water table in Fig. 10) shows that in the presence of coarse sand, the pipe drains a greater water volume from the inner part of the beach, which is not useful for BDS aims. The main aim is, in fact, to desaturate the submerged seaward-sided beach. First, the results allow to state that with high value of permeability the drain is useless and the beach can be considered naturally drained. 


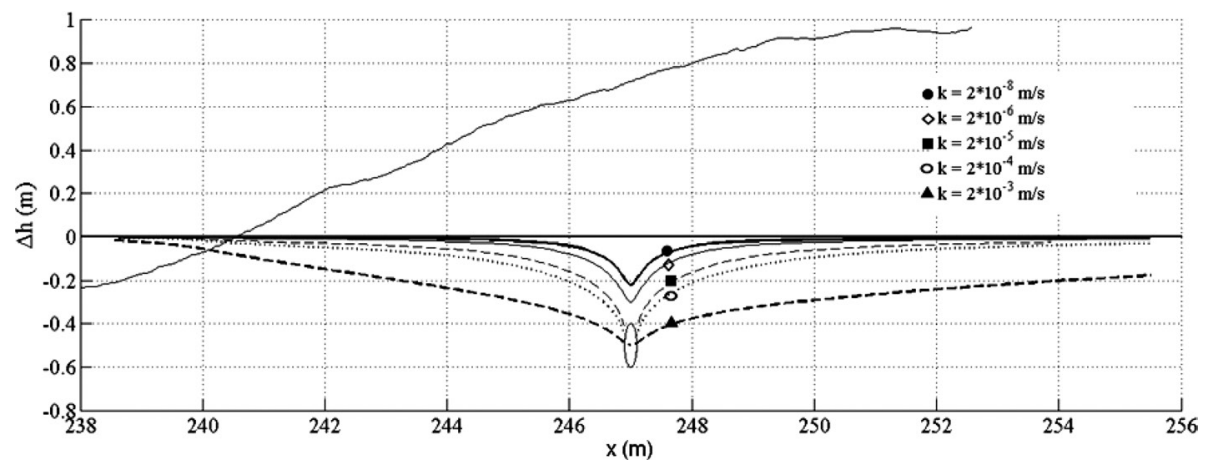

Figure 10: Numerical spatial variation of water table lowering with different beach permeabilities (Test S1 - drain D3).

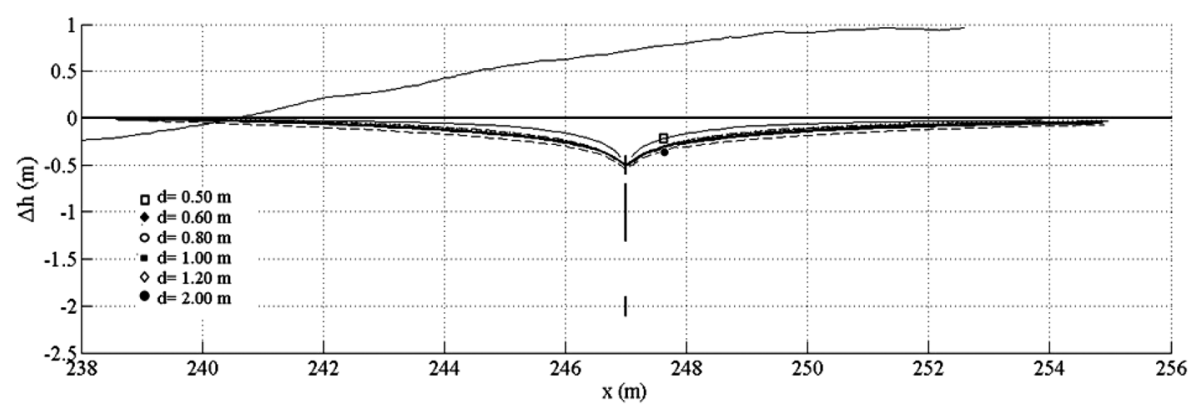

Figure 11: Numerical spatial variation of water table lowering with different drain depths (Test S1 - Drain D3) in a beach with a permeability equal to $2 \times 10^{5} \mathrm{~m} / \mathrm{s}$.

BDS effects on water table lowering have been also simulated with different drain depths with respect to the undisturbed water table. Figure 11 shows the numerical spatial variation of water table lowering for the drain D3 placed in a beach with permeability equal to $2 \times 10^{5} \mathrm{~m} / \mathrm{s}$ for different drain depths (from 0.5 to $2.00 \mathrm{~m}$ below the undisturbed water table).

When the drain depth varies in a range between 0.5 and $0.8 \mathrm{~m}$, it can be observed that the maximum water table lowering and, consequently, the unsaturated area, increase with drain depth. In such a situation, drain seems to work in free surface flow condition. Below $0.8 \mathrm{~m}$, the maximum water table lowering remains quite constant with the increase of drain depth, probably due to the fact that the drain begins to work in pressure. With a 2-m deep drain, the maximum water table lowering is almost the same that of one produced by a drain placed at $1.20 \mathrm{~m}$ with a little increase in the unsaturated area. As a consequence, it can be stated that a drain deeper than $0.60 \div 0.80 \mathrm{~m}$ would be more expensive with no advantages in terms of drainage system efficacy. This 'limit drain depth' is strongly influenced by beach grain characteristics. In fact, Figure 12 reports the water table lowering induced by a drain placed at different depths in a beach with permeability equal to $2 \times 10^{3} \mathrm{~m} / \mathrm{s}$. In a beach characterized by a higher value of permeability, a free surface flow occurs up to a drain depth equal about to $1.20 \mathrm{~m}$. In this case, the 'limit drain depth' previously defined can be set at about $1.50 \mathrm{~m}$. Analysing all simulated permeabilities, it can be observed that the decrease in beach permeability corresponds to a 'limit drain depth' reducing. This limit can be, 


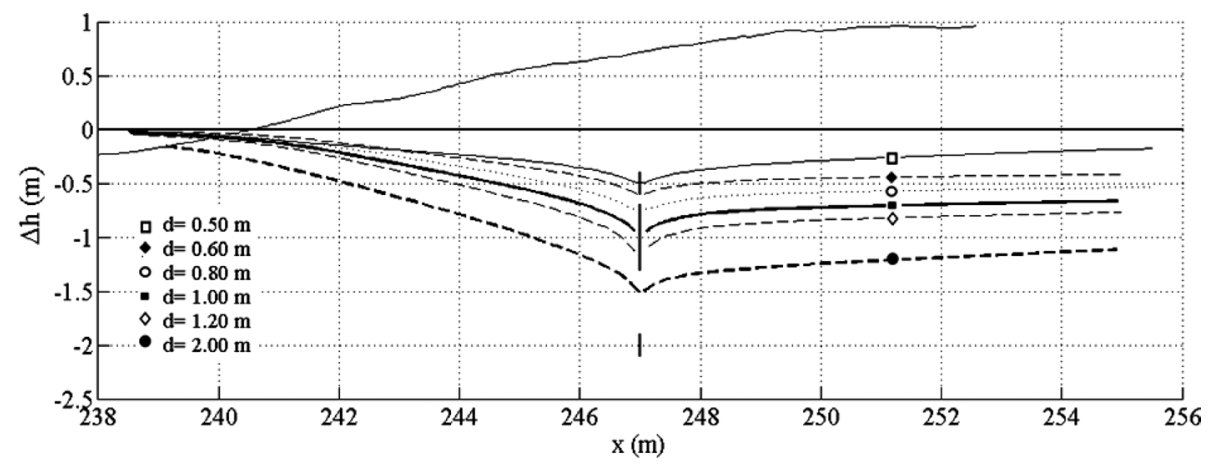

Figure 12: Numerical spatial variation of water table lowering at different drain depths (Test S1 Drain 3 on) in a beach with a permeability equal to $2 \times 10^{3} \mathrm{~m} / \mathrm{s}$.

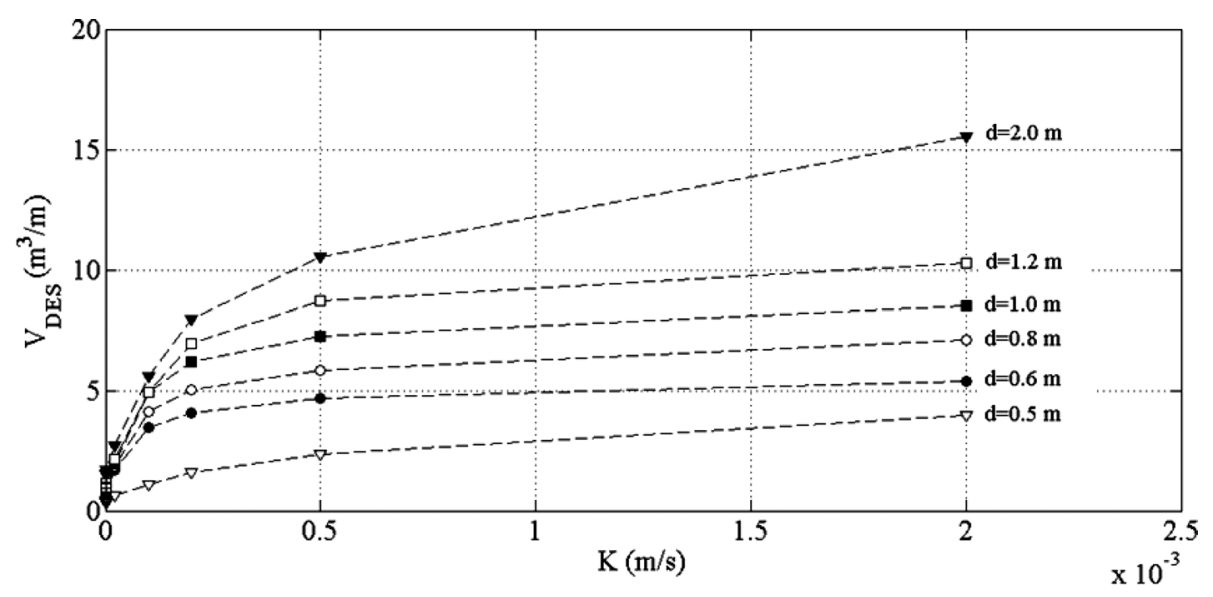

Figure 13: Calculated desaturated beach volume for unit of length $\left(V_{D E S}\right)$ vs. permeability $(K)$.

therefore, defined as the maximum drain depth, related to the beach permeability, which produces the maximum water table lowering (and unsaturated area) allowing a free surface flow within the drain pipe.

In Figure 13, the calculated desaturated beach volume $V_{D E S}$ for unit of length is reported as a function of permeability $K$. Each curve refers to different drain depths. As expected, the desaturated beach volume increases with the increase in both permeability and drain depth. In correspondence to high values of permeability, the desaturated volume is more influenced by the increased drain depth. In correspondence to lower value of permeability, the drainage process is mainly influenced by beach characteristics rather than drain altimetric position.

The knowledge of beach volume that the system is able to dry is necessary in order to know the water flows drained by the system and, consequently, to design the drain pipe, and, in particular the drain diameter.

Figure 14 reports the drained flows $Q$ versus the desaturated beach volume $V_{D E S}$ both calculated for unit of length, related to different drain depths. Each curve refers to different drain depths and 


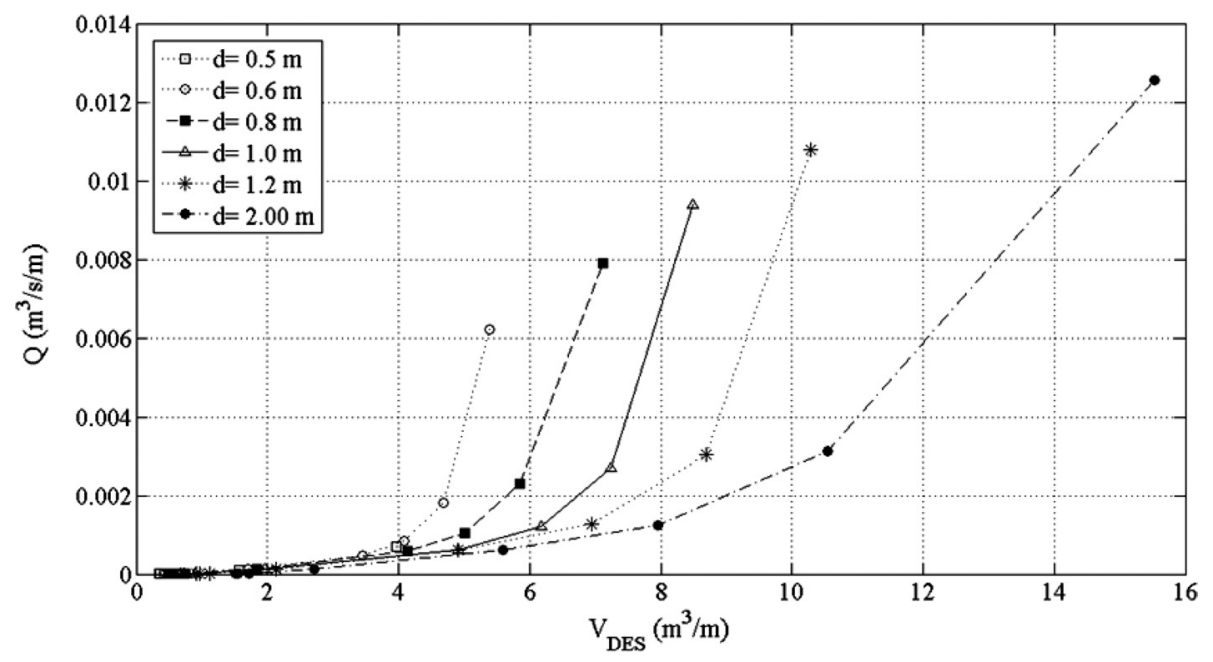

Figure 14: Calculated drained flows $(Q)$ vs. calculated desaturated beach volume $\left(V_{D E S}\right)$ for unit of length.

it is made from points related to different values of permeability. Previously, Fig. 13 shows that an increase in permeability corresponds to an increase in desaturated volume. Consequently, in Fig. 14 , moving from the left to the right over a curve, each value corresponds to an increase in the permeability.

The drained flows increase with the desaturated volume and, consequently, with permeability for all simulated drain depths. Similarly, the drained flow increases with drain depth. Figure 14 allows to observe that with small values of $V_{D E S}$, which correspond to small values of permeability, drained flows are very low and the drain depth does not have a great influence on the drained flows. As stated before, the infiltration processes are mainly governed by beach characteristics. With higher values of permeability, the influence of drainage system begins to be important. Such a phenomenon is confirmed by the remarkable increase of drained flows in correspondence to higher value of permeability at deeper drain position. Moreover, obtained data show that for each drain depth (except for a 2.00-m deep drain) a limit value of permeability can be identified. In correspondence to this value (ranging from $2 \times 10^{4}$ to $2 \times 10^{3} \mathrm{~m} / \mathrm{s}$ ), a small increase in $V_{D E S}$ can produce a much higher increase of drained flow, which would be useless and would cause an increased installation costs, without any advantage in reducing the saturation degree of the beach.

\section{CONCLUSIONS}

The reported simulations represent the first step for a more complete numerical modelling of the phenomena interested in a drainage process.

Richard's equation, numerically solved by the HYDRUS-2D code, is used in order to reproduce the infiltration processes in a drained beach in static conditions (without waves). Such an analysis allows stating some first considerations about BDS design guidelines.

Numerical simulations first aim to validate the code, through comparing numerical data with experimental ones. The comparison between numerical and experimental spatial variation of the local hydraulic head during the drainage process shows a good agreement. Some differences between numerical simulations and experiments can be found in the analysis of the time variation of water 
table lowering. The transient process simulated by the code is more gradual than that one, which occurs during experiments and reaches the quasi-steady state after about $30 \mathrm{~min}$. Conversely, during experiments, it is observed that about the $70 \div 80 \%$ of the maximum water table lowering occurs in the first 8-10 min, reaching the quasi-stationary condition after about $10 \mathrm{~min}$. Moreover, the data show that the measured and calculated drained flows are comparable.

Such a good comparison allows running other numerical simulations of the drainage system applications, in order to draw, in static condition, first system design considerations.

Drainage processes are first analysed in a beach characterized by different values of permeability. The values of permeability simulated range from $2 \times 10^{3} \mathrm{~m} / \mathrm{s}$ (coarse sand) to $2 \times 10^{8} \mathrm{~m} / \mathrm{s}$ (silt). As expected, the increase in permeability causes an increase in maximum water table lowering in correspondence to the working drain and, as a consequence, an increase in the unsaturated area below the undisturbed water table inside the beach can be observed. With greater value of permeability (typical of gravel), the drainage system is useless, because the beach can be considered naturally drained. No advantages would be obtained by adding an artificial drainage. On the contrary, sand too much fine would nullify the drainage efficiency.

System response is also simulated considering different drain depths (from 0.5 to $2.00 \mathrm{~m}$ ) from the undisturbed water table level, under constant permeability conditions. The deeper drain altimetric position favours the increased desaturated beach. The analysis allows to define a 'limit drain depth' that highly depends on beach permeability. It can be identified as the maximum drain depth, in correspondence to which the water table reaches its maximum. In general, it corresponds to the depth at which the drain pipe should begin to work in pressure. Consequently, a drain placed below such a defined limit would not cause any positive effects on system efficiency with an increase in installation costs. Desaturated volumes for unit of length $\left(V_{D E S}\right)$ are analysed as a function of permeability for different drain depths. Data show that the efficiency in draining the beach increases with both the permeability and the drain depth. Data show that in a beach characterized by low values of $K$, the system response is highly influenced by the beach permeability. With higher values of permeability, the altimetric drain position increasingly influences the desaturated beach volumes, which depend on both beach and drain characteristics.

The drainage system design also regards the pipe dimensions and, in particular, the drain diameter. To have the best BDS efficacy, it is important to determine the water volume that is intended to be drained. For this purpose, drained flows are analysed as a function of desaturated volumes. The analysis shows that the drained flows increase with both the $V_{D E S}$ and the permeability and allows to confirm that with low values of permeability, infiltration processes are mainly governed by beach characteristics. The influence of drain depth with respect to the SWL begins to be important in correspondence with higher value of permeability. Moreover, obtained data show that for each drain depth (except for a 2.00-m deep drain), a limit value of permeability can be identified, ranging from $2 \times 10^{4}$ to $2 \times 10^{3} \mathrm{~m} / \mathrm{s}$, in correspondence to which a small increase in $V_{D E S}$ can produce a high increase in drained flow without any advantage in reducing the saturation degree of the beach and, consequently, useless for BDS purposes.

The present work represents a first step in BDS modelling and designing. Once this process will be numerically well understood, the simulations will be completed with the other two macro processes regarding the external wave motion and the sediment transport in the swash zone. Future applications mainly regard the possibility of improving the adopted numerical model by simulating the drainage efficiency under different wave attacks in order to analyse in deep the influence of wave energy on groundwater behaviour and how this interaction can produce a reduction in the off-shore sediment transport. 


\section{REFERENCES}

[1] Charlier, R.H. \& De Meyer, C.P., Coastal Erosion: Response and Management, Springer-Verlag: Berlin, Heidelberg and New York, 1998, ISBN: 3-540-60022-1.

[2] Chappell, J., Eliot, I.G., Bradshaw, M.P. \& Lonsdale E., Experimental control of beach face dynamics by water table pumping. Engineering Geology, 14(1), pp. 29-41, 1979. doi: http:// dx.doi.org/10.1016/0013-7952(79)90061-9

[3] Rossetti, R., Damiani, L. \& Ranieri, G., Coastal protection with BMS: the first experience in Italy. Coastal Engineering, 6, pp. 365-376, 2003.

[4] Bowman, D., Ferri, S. \& Pranzini, E., Efficacy of beach dewatering - Alassio, Italy. Coastal Engineering, 54, pp. 791-800, 2007. doi: http://dx.doi.org/10.1016/j.coastaleng.2007.05.014

[5] Ciavola, P., Fontana, E. \& Vicinanza, D., Performance of a beach drainage system at Lido Adriano (Ravenna, It aly), Proceedings of 33rd IAHR Congress: Water Engineering for a sustainable environment, British Columbia, Vancouver, pp. 7312-7319, 2009.

[6] Elfrink, B. \& Baldock, T., Hydrodynamics and sediment transport in the swashzone: a review and perspectives. Coastal Engineering, 45(3-4), pp. 149-167, 2002. doi: http://dx.doi. org/10.1016/s0378-3839(02)00032-7

[7] Larson, M., Kubota, S. \& Erikson, L., Swash-zone sediment transport and foreshore evolution: field experiments and mathematical modelling. Marine Geology, 212(1-4), pp. 61-79, 2004. doi: http://dx.doi.org/10.1016/j.margeo.2004.08.004

[8] Bagnold, R.A., Beach formation by waves: some model experiments in a wave tank, Journal of the ICE, 15(1), pp. 27-52, 1940. doi: http://dx.doi.org/10.1680/ijoti.1940.14279

[9] Turner, I.L. \& Masselink, G., Swash infiltration-exfiltration and sediment transport. Journal of Geophysical Research, 103(C13), pp. 30813-30824, 1998. doi: http://dx.doi. org/10.1029/98jc02606

[10] Robinson, C., Gibbes, B. \& Li, L., Driving mechanisms for groundwater flow and salt transport in a subterranean estuary. Geophysical Research Letters, 33(3), L03402, 2006. doi: http:// dx.doi.org/10.1029/2005g1025247

[11] Grant, U.S., Effects of groundwater table on beach erosion. Bulletin of the Geological Society of America, 57, 1946.

[12] Grant, U.S., Influence of the water table on beach aggradation and degradation. Marine Research, 7(3), pp. 655-660, 1948.

[13] John, R. \& Duncan, Jr., The effects of water table and tide cycle on swash-backwash sediment distribution and beach profile development. Marine Geology, 2(3), pp. 186-197, 1964. doi: http://dx.doi.org/10.1016/0025-3227(64)90039-8

[14] Machemehl, J.L., French, T.J. \& Huang, N.E., New method for beach erosion control. Proceedings of Civil Engineering in the Oceans, 1, pp. 142-160, 1975.

[15] Kawata, Y. \& Tsuchiya, Y., Applicability of sub-sand system to beach erosion control, Proceedings of the International Conference on Coastal Engineering, 1(20), 1986.

[16] Oh, T.M. \& Dean, R.G., Beach face dynamics as affected by ground water table elevations, University of Florida, Department of Coastal and Oceanographic Engineering, 1992.

[17] Masselink, G., Turner, I.L. \& Williams, J.J., Large-scale laboratory investigation into the effect of the beach ground water table on gravel beach morphology. Journal of Coastal Research, SI(56), pp. 93-97, 2009.

[18] Vesterby, H., Beach face dewatering: the European ex perience, Proceedings of 7th National Conference on Beach Preservation Technology, pp. 53-68, Tampa, Florida, 1994. 
[19] Damiani, L., Petrillo, A.F. \& Saponieri, A., Beach dewatering systems: modelling coastal groundwater flow, Proceedings of 33rd IAHR Congress: Water Engineering for a sustainable environment, pp. 7304-7311, British Columbia, Vancouver, 2009.

[20] Damiani, L., Aristodemo, F., Saponieri, A., Verbeni, B., Veltri, P. \& Vicinanza, D., Full scale experiments on a BDS: hydrodynamic effects inside the beach. Journal of Hydraulic Research, 49(S1 Hydralab III), pp. 44-54, 2011, ISSN: 0022-1686. doi: http://dx.doi.org/10.1080/002 21686.2011.588515

[21] Aristodemo, F., Ciavola, P., Veltri, P. \& Saponieri A., The influence of a beach drainage system on wave reflection and surf beat processes. Journal of Coastal Research, SI 64, pp. 455-459, 2011, ISSN: 0749-0208.

[22] Damiani, L., Vicinanza, D., Aristodemo, F., Saponieri, A. \& Corvaro, S., Experimental investigation on wave set-up and nearshore velocity field in presence of a BDS. Journal of Coastal Research, SI 64, pp. 55-59, 2011, ISSN: 0749-0208.

[23] Saponieri, A. \& Corvaro, S., Analisi sperimentale di una spiaggia in presenza di un sistema di drenaggio. Studi Costieri, 20, pp. 85-93, 2012, ISSN: 1129-8588.

[24] Contestabile, P., Aristodemo, F., Vicinanza, D. \& Ciavola, P., Laboratory study on a beach drainage system. Coastal Engineering, 66, pp. 50-64, 2012. doi: http://dx.doi.org/10.1016/j. coastaleng.2012.03.012

[25] Packwood, A.R., The influence of beach porosity on wave uprush and backwash. Coastal Engineering, 7(1), pp. 29-40, 1983. doi: http://dx.doi.org/10.1016/0378-3839(83)90025-x

[26] Damiani, L. \& Ranieri, G., Contributo allo studio delle oscillazioni della linea di riva. Atti e relazioni, Accademia Pugliese delle Scienze, 45(2), pp. 93-119, 1988.

[27] Kobayashi, N. \& Wurjanto, A., Numerical model for waves on rough permeable slopes. Journal of Coastal Research, pp. 149-166, 1990.

[28] Nielsen, I., Tidal dynamics of the water table in beaches. Water Resources Research, 26(9), pp. 2127-2134, 1990. doi: http://dx.doi.org/10.1029/wr026i009p02127

[29] Li, L., Barry, D.A. \& Pattiaratchi, C.B., Modeling coastal ground-water response to beach dewatering. Journal of Waterway, Port, Coastal and Ocean Engineering ASCE, 122(6), pp. 273-280, 1996. doi: http://dx.doi.org/10.1061/(asce)0733-950x(1996)122:6(273)

[30] Li, L., Barry, D.A. \& Pattiaratchi, C.B., Numerical modelling of tide-induced beach water table fluctuations. Coastal Engineering, 30, pp. 105-123, 1997. doi: http://dx.doi.org/10.1016/ s0378-3839(96)00038-5

[31] Vesterby, H., Mangor, K. \& Refsgaard, A., The beach drainage concept: development of an engineering design tool. Proceedings of COPEDEC V, 2, pp. 961-971, 1999.

[32] Li, L., Barry, D.A., Pattiaratchi, C.B. \& Masselink, G., Beachwin: modelling groundwater effects on swash sediment transport and beach profile changes. Environmental Modelling Software, 17(3), pp. 313-320, 2002. doi: http://dx.doi.org/10.1016/s1364-8152(01)00066-4

[33] Ioannidis, D. \& Karambas, Th.V., 'Soft' shore protection methods: beach drainage system. Proceedings of International Conference on Environmental Science and Technology, 10, pp. 528-535, 2007.

[34] Feddes, R.A., Kowalik, P.J. \& Zaradny, H.., Simulation of Field Water Use and Crop Yield, Wiley, 1978.

[35] van Genuchten, Th., A numerical model for water and solute movement in and below the root zone. Research Report 121, U.S. Salinity Laboratory, USDA, ARS, Riverside, CA, 1987.

[36] van Genuchten, Th., A closed-form equation for predicting the hydraulic conductivity of unsaturated soils. Soil Science Society of America Journal, 44(5), pp. 892-898, 1980. doi: http://dx.doi.org/10.2136/sssaj1980.03615995004400050002x 
[37] Mualem, S., A new model for predicting the hydraulic conductivity of unsaturated porous media. Water Resources Research, 12(3), pp. 513-522, 1976.

[38] Neuman, S.P., Feddes, R.A. \& Bresler, E., Finite element simulation of flow in saturated and unsaturated soil considering water uptake by plants. Third Annual Report, Hydraulic Engineering Lab., Haifa, Israel, 1974.

[39] Feddes, R.A., Bresler, E. \& Neuman, S.P., Field test of a modified numerical model for water uptake by root systems. Water Resources Research, 10(6), pp. 1199-1206, 1974. doi: http:// dx.doi.org/10.1029/wr010i006p01199

[40] Neuman, S.P., Galerkin approach to saturated-unsaturated flow in porous media. Finite Elements in Fluid, 1, 1975.

[41] Pinder, G.F., Finite Element Simulation in Surface and Subsurface Hydrology, 1st edn., Academic Publishers, 1977.

[42] Saponieri, A. \& Damiani, L., Groundwater flow in a drained beach. WIT Transactions on Modelling and Simulation, 55, 2013. doi: http://dx.doi.org/10.2495/cmem130201 